\title{
A Simulation-Based Nonlinear Goal Programming Model for Groundwater Remediation Systems Design
}

\author{
Jing $\mathrm{Li}^{1}, \mathrm{Li} \mathrm{He}^{1,2 *}$, Hongwei $\mathrm{Lu}^{1,2}$, Min $\mathrm{Xu}^{3}$ \\ 'Resources and Environmental Research Academy, North China Electric Power University, Beijing 102206, China \\ ${ }^{2}$ College of Renewable Energy, North China Electric Power University, Beijing 102206, China \\ ${ }^{3}$ Water Environment Institute, Chinese Academy for Environmental Planning, Beijing 100012, China
}

Received: 9 May 2014

Accepted: 3 August 2014

\begin{abstract}
This study proposes an integrated method that simulates and optimizes groundwater design and management in combination with goal programming, which establishes the equilibrium between technical and environmental constraints in a pump-and-treat system. This method is applied to a petroleum-contaminated site in Western Canada to identify optimal remediation strategies given a set of remediation scenarios. The significant influential factors are remediation duration, standard concentration levels, and total pumping rate. Results indicate that goal programming can greatly enhance the remediation effect under low contaminant concentrations. In the pump-and-treat system, wells I2, E1, and E3 are the dominant components, whereas wells M7 and M5 are sensitive to variations in the identified influential factors. These wells must therefore be monitored intentionally. Moreover, these factors influence one another in interaction. Thus, high total pumping rates do not always generate favorable outcomes, and a long remediation period is unnecessary. In conclusion, the three identified factors should be spontaneously considered in the general goal-programming framework.
\end{abstract}

Keywords: groundwater remediation, goal programming, optimal design, pump-and-treat systems

\section{Introduction}

Pump-and-treat (PAT) techniques are often applied to the remediation of dissolved chemicals in aquifers [1-3]. Among these chemicals, contaminants from petroleum products are widespread in groundwater because of leakage from underground storage tanks and pipelines in numerous sites [4]. Therefore, petroleum pollution in groundwater is an important concern, and many researchers aim to develop appropriate techniques [5]. PAT is the best-understood remediation technology and is most widely used to clear the contaminated plumes in aquifers because of the simple devices used in the process and its pronounced mass

*e-mail: li.he@ncepu.edu.cn removal of contaminants. To minimize remediation cost, the relevant management problem typically emphasizes the optimal selection of an appropriate pumping policy while simultaneously meeting a set of technical, economic, and social constraints [6]. This management problem is thus addressed through the simulation of contaminant concentration models in combination with optimization algorithms [7].

Much research has been conducted on PAT system design optimization. Chang et al. [8] proposed a dynamic PAT groundwater remediation system that applies both the genetic algorithm and constrained differential dynamic programming. The system design can accommodate the optimal number and locations of wells. Matott et al. [9] conducted a similar study to examine the analytical flow mod- 
els of the element method in a PAT containment system. The study results show that these models effectively optimize the locations, number, and pumping rates of wells. The number and locations of wells are definite at most petroleum-contaminated sites because these wells existed prior to groundwater design and management. Moreover, the design and management of PAT remediation systems for contaminated aquifers are often strongly and unpredictably influenced by factors such as subsurface investigation programs. Bau and Mayer [10] developed a stochastic data-worth framework to estimate the economic opportunity of such programs. The results suggest that the value of information is relevant to the degree of risk aversion and the cleanup target violation of decision makers. In addition, they generally rely on groundwater simulation and optimization techniques to ensure the safety of groundwater quality. Therefore, the crucial objectives of groundwater design and management include simulating the fate forecasting of contaminants in the subsurface and the optimization of groundwater remediation.

However, poor computational efficiency is a common problem in groundwater simulation and optimization [11, 12]. Therefore, researchers develop advanced methods to strengthen computational efforts [13-15]. Effective surrogates can be generated to appropriately replace complex simulation equations. In line with this remedy, an adaptive neural network algorithm was introduced in [16] to save many of the simulation model calls; however, the accuracy of this algorithm lacks comparability. Nonetheless, this method can significantly reduce the computational efforts in the optimal programming of large-scale water resources. Bau and Mayer [17] also proposed a surrogate form of the objective function to lower very high computational cost in the stochastic management of PAT strategies [17]. Moreover, the simulation surrogates are examined through stepwise quadratic response surface analysis to realize automatic variable selection and surrogate development. In particular, this method can predict the flow and transport of petroleum contaminants over three-dimensional aquifers [18].

The total cost of a PAT system is deemed to be the most significant objective [19]. However, the final optimization is influenced by several factors in practice, including environmental and technological constraints. Therefore, an appropriate method is combined with simulation and optimization to accurately establish the relationships among these influential factors. In line with this objective, goal programming enables decision-makers to consider several objectives simultaneously in the determination of a set of acceptable solutions. Bravo and Gonzalez [20] applied stochastic goal programming to study a water-use planning case. The agency in this decision model establishes two parallel goals, namely farm management and environmental effect. Furthermore, the agency allows irrigation of the available surface water, standard groundwater, and complementary groundwater. Furthermore, Nidumolu et al. [21] developed an interactive, multi-goal linear programming model that merges with an inter-stakeholder communication matrix. Multiple stakeholders are considered objectives in this model. Moreover, the stakeholder communication matrix is analyzed through scenarios to gain insight into the interactions among stakeholders and to take the necessary curative measures to improve communication.

All of the aforementioned studies indicate that goal programming can be practical in decision-making given several expected objectives [22-24]. Nonetheless, few studies apply goal programming to the PAT system, although it is the most widely used method of groundwater treatment. Consequently, this study proposes an integrated method of simulation and optimization for groundwater design and management that is integrated with goal programming. Throughout the entire process of simulation and optimization, three main tasks are completed:

i) The simulation and prediction of the behavior of the contaminants in aquifers

ii) The improvement of computational efficiency through stepwise quadratic response surface analysis to address the complex nonlinear relationships among the models

iii) The identification of optimal remediation strategies under various scenarios through goal programming

\section{Methods}

\section{Primary Optimization Model}

This study aims to establish an optimization model that bridges the decision variables in the PAT system and its total cost. Contaminated water is extracted to the surface through extraction wells, whereas treated water is injected underground through injection wells [25]. Thus, the extraction and injection rates of the extraction and injection wells are variables in the PAT system. Previous studies report that operating cost dominates total cost [26]. A high proportion of operating cost is in turn composed of extraction and injection rates. Therefore, total pumping rate is set as an objective of this model. This rate is a summation of all of the extraction and injection rates of the extraction and injection wells.

The model constraints come in two forms: environmental and technological constraints. In the environmental constraints, contaminant concentration should be less than the regulated environmental standard. Thus the monitoring wells of the PAT system are used to manage contaminant concentrations in a contaminated region; these concentrations must meet the environmental standard in all monitoring wells. In the technological constraints, the extraction and injection rates should be limited to within a given range of lower and upper bounds. In addition, the sum of the extraction rates in all extraction wells should be equal to that of the injection rates in all injection wells. The primary optimization model in the PAT system can therefore be formulated as:

$$
M i n=\sum_{i}^{m} Q_{E i}+\sum_{j}^{n} Q_{l j}
$$

s.t

$$
C_{k} \leq C_{s t a}
$$




$$
\begin{gathered}
\sum_{i}^{m} Q_{E i}=\sum_{j}^{n} Q_{l j} \\
Q_{\text {lower }} \leq Q_{E i} \leq Q_{\text {upper }} \\
Q_{\text {lower }} \leq Q_{l j} \leq Q_{\text {qpper }} \\
i=1,2, \ldots m \\
j=1,2, \ldots n \\
k=1,2, \ldots l
\end{gathered}
$$

...where $Q_{E i}$ represents the extraction rate in the $i$ th extraction well; $Q_{l j}$ denotes the injection rate in the $j$ th injection well; $C_{k}$ is the predicted contaminant concentration in the $k$ th monitoring well; $C_{s t a}$ is the given environmental standard; $Q_{\text {lower }}$ and $Q_{\text {upper }}$ are the lower and upper bounds of the pumping rate, respectively; and $m, n$, and $l$ are the numbers of extraction, injection, and monitoring wells, respectively.

\section{Simulation Model and Surrogates}

In the process of simulation and optimization, the first critical step is to predict contaminant concentration [27]. Thus, a three-dimensional, multiphase, and multicomponent simulation model is proposed. The basic mass conservation equation for the subsurface components can be written as:

$$
\frac{\partial}{\partial t}\left(\phi \widetilde{C}_{k} \rho_{k}\right)+\vec{\nabla} \cdot\left[\sum_{l=1}^{n_{p}} \rho_{k}\left(C_{k l} \vec{u}_{l}-\phi S_{l} \overrightarrow{\vec{D}}_{k l} \cdot \vec{\nabla} C_{k l}\right)\right]=R_{k}
$$

...where $k$ is the component index, $l$ is the phase index, $\varnothing$ is porosity, $\widetilde{C}_{k}$ is the overall concentration of component $k$ (volume of component $k$ per unit pore volume), $\rho_{\mathrm{k}}$ is the density of component $k\left[\mathrm{ML}^{-3}\right], n_{p}$ is the number of phases, $C_{k l}$ is the concentration of component $k$ in phase $l$ (volume fraction), $\vec{u}_{l}$ is the Darcy velocity of phase $l\left[\mathrm{LT}^{-1}\right], S_{l}$ is the saturation of phase $l$ (volume of phase $l$ per volume of pores), $R_{k}$ is total source/sink term for component $k$ (volume of component $k$ per unit volume of porous media per unit time), and $\widetilde{C}_{k}$ is volume of component $k$ as summed up across all phases.

During simulation, the conservation equations can be derived by summing up the mass balances for each constituent across all phases. The pressure equation is generated by adding up the mass balance equations of the overall volume-occupying components. In response, $C_{k}$ represents the contaminant concentration in the kth monitoring well. $C_{k}$ is closely connected with the decision variables (extraction and injection rates) and can thus be regarded as a polynomial of these variables as obtained using the surrogate equations. This surrogate can be formulated as:

$c_{k}=a_{0, k}+\sum_{i=1}^{n} a_{i, k} Q_{i}+\sum_{i=1}^{n} \sum_{j=1}^{n} a_{i j, k} Q_{i} Q_{j}(i \neq j)+\sum_{i=1}^{n} a_{i i, k} Q_{i}^{2}+e_{k}$

...where $a_{0 k}$ is the intercept term of surrogate $k ; \sum_{i=1}^{n} a_{i, k} Q_{i}$ are the linear terms of surrogate $k ; \sum_{i=1}^{n} \sum_{j=1}^{n} a_{i j, k} Q_{i} Q_{j}(i \neq j)$ are the interaction terms of surrogate $k ; \sum_{i=1}^{n} a_{i i, k} Q_{i}^{2}$ are the quadratic terms of surrogate $k ; e_{k}$ is the error of surrogate $k$; and $n$ is the number of explanatory variables.

The contaminant concentrations are related to the operating conditions through stepwise quadratic response surface analysis. This technique can simplify the surrogate with statistically insignificant and unnecessary terms. It can also increase the accuracy of the surrogate because some of the statistically significant terms are arbitrarily neglected. In comparison with existing approaches, the stepwise quadratic response surface analysis displays (1) enhanced capability to address discontinuous variables along with the nonlinear bridge among the variables, and (2) improved suitability for the identification of specific relationships between the explanatory variables and the response variables when available information is limited [28].

\section{Goal Programming Model}

The first goal of the design of the groundwater PAT system concerns the total pumping rate related to operating cost, whereas the second goal involves the environmental and technological constraints. Thus, goal programming is introduced to determine an optimal solution that meets all of the goals. The total pumping rate should be less than a certain upper bound in the first goal. To reduce cost given the limited budget in real-world engineering, Formula (1a) can be rewritten as:

$$
\begin{aligned}
& T R=\sum_{i}^{m} Q_{E i}+\sum_{j}^{n} Q_{I j} \\
& T R+\widehat{S}^{-}-\widehat{S}^{+} \leq T R_{\max }
\end{aligned}
$$

...where $T R$ is the total pumping rate, which is the sum of all extracting and injecting rates; $T R_{\max }$ is the given total pumping rate; $\widehat{s}^{+}$indicates that the excess of TR is higher than $T R_{\max }$; and $\widehat{s}^{-}$suggests that the excess of $T R_{\max }$ is higher than $T R$.

The contaminant concentrations in all monitoring wells should be limited to within the environmental standard in the first part of the second goal. As expected, the contaminant concentration must be minimized. In actual practice, the contaminant concentrations in all monitoring wells may not always accord with environmental standards. Thus, a set of variables $\left(\widehat{d}_{k}^{-}\right.$and $\left.\widehat{d_{k}^{+}}\right)$is introduced to represent the residual quantity between the environmental standard and the actual concentration. $\widehat{d_{k}^{+}}$denotes the level at which actual concentration is higher than the environmental standard, whereas $\widehat{d}_{k}^{-}$corresponds to the level at which actual concentration is lower than environmental standard. Formula (1b) can then be rewritten as:

$$
C_{k}+\widehat{d}_{k}^{-}-\widehat{d}_{k}^{+} \leq C_{s t a}
$$

The other part of the second goal concerns the balance between the total extraction and total injection rates. In realworld engineering, the absolute equilibrium is difficult to 
achieve. To simulate actual situations effectively, $\widehat{r}^{+}$and $\widehat{r}^{-}$ are introduced to describe the difference between the total extraction and the total injection rates. Formula (1c) can then be expressed as

$$
\sum_{i}^{m} Q_{E i}-\sum_{j}^{n} Q_{I j}-\widehat{r}^{+}+\widehat{r}^{-}=0
$$

...where $\widehat{r}^{+}$represents the level at which the total extraction rate is higher than the total injection rate and $\hat{r}^{-}$indicates the level at which the total extraction rate is lower than the total injection rate.

Accordingly, the objective of the basic optimization model is replaced as a constraint. The other constraints are rewritten as previously mentioned. The model is then converted to identify the most ideal remediation solution state when all costs are met along with the environmental and technological goals. The levels of $\widehat{d}_{k}^{-} / \widehat{d}_{k}^{+}, \widehat{r}^{+} / \widehat{r}^{-}$, and $\widehat{s}^{+} / \widehat{s}^{-}$ may indicate the distance between the optimal remediation solution and this ideal state. However, $\widehat{d}_{k}^{-} / \widehat{d}_{k}^{+}, \widehat{r}^{+} / \hat{r}^{-}$, and $\hat{s}^{+} / \hat{s}^{-}$correspond to different meanings and influences on various units. The statistical weights of $\widehat{d}_{k}^{-} / \widehat{d}_{k}^{+}, \widehat{r}^{+} / \hat{r}^{-}$, and $\widehat{s}^{+} / \widehat{S}^{-}$are thus introduced to unify these variables into one equation. Accordingly, the model can be rewritten as:

$$
\text { Max }=\sum_{k}^{l} x_{k} \cdot \widehat{d}_{k}^{-}+x_{k+1} \cdot \widehat{s}^{-}-x_{k+2} \cdot \widehat{r}^{+}
$$

s.t.

$$
\begin{gathered}
T R=\sum_{i}^{m} Q_{E i}+\sum_{j}^{n} Q_{I j} \\
T R+\widehat{s}^{-}-\widehat{s}^{+} \leq T R_{\max } \\
\hat{s}^{+} \cdot \widehat{s}^{-}=0 \\
C_{k}=f_{k}\left(Q_{E i}, Q_{l j}\right) \\
C_{k}+\widehat{d}_{k}^{-}-\bar{d}_{k}^{+} \leq C_{\text {sta }} \\
\hat{d}_{k}^{+} \cdot \widehat{d}_{k}^{-}=0 \\
\sum_{i}^{m} Q_{E i}-\sum_{j}^{n} Q_{I j}-\hat{r}^{+}+\widehat{r}^{-}=0 \\
\hat{r}^{+} \cdot \widehat{r}^{-}=0 \\
Q_{\text {lower }} \leq Q_{E i} \leq Q_{\text {upper }} \\
Q_{\text {lower }} \leq Q_{l j} \leq Q_{\text {upper }} \\
i=1,2, \ldots m \\
j=1,2, \ldots n \\
k=1,2, \ldots l
\end{gathered}
$$

...where $x_{k}$ is the set weight of $\widehat{d}_{k}^{-} ; x_{k+1}$ is the set weight of $\tilde{s}^{-} ; x_{k+2}$ is the set weight of $\widehat{r}^{+}$; and $C_{k}$ is the function of $Q_{E i}$ and $Q_{I j}$. The three pairs of variables $\left(\widehat{d}_{k}^{-} / \widehat{d}_{k}+, \widehat{r}^{+} / \widehat{r}^{-}\right.$, and $\left.\widehat{s}^{+} / \widehat{s}^{-}\right)$ are bound under a mutual exclusivity constraint. When the sign of a variable is positive, the exclusive variable must be zero. Thus, the product of the three pairs of variables is always zero. In the practical PAT system, a high level of $\widehat{d}_{k}^{-}$ suggests that the predicted concentration is significantly lower than the given environmental standard after a period of remediation. A high $\widetilde{s}^{-}$level indicates that the predicted total pumping rate is significantly lower than a given lowcost total pumping rate. However, a low $\widehat{r}^{+}$level suggests that as expected, the total extraction and total injection rates are close to equilibrium. Therefore, the objective formula involves the maximum values of positive $\widehat{d}_{k}^{-}$and $\widehat{s}^{-}$, in which $\widehat{s}^{-}$is negative.

\section{Results and Discussion}

\section{Study Site}

The developed model is applied to a petroleum-contaminated aquifer in western Canada. A gas plant in the west and a disposal pit in the northeast are identified as the two contamination sources, and the groundwater flows southwest. The site houses complex soil types, including gravel, clay, tills, and silty clay. The contaminant plume was once surrounded by two injection and four extraction wells. Eight monitoring wells are drilled to check contaminant concentrations, which are subject to an environmental standard. All these wells are subject to PAT treatment, and their distribution is shown in Fig. 1. The contaminated domain of the site measures $130 \times 120$ and is $10 \mathrm{~m}$ deep. The area is discretized into $26 \times 24$ grid blocks, and depth is segmented into four layers. Each grid has dimensions of 5, 5, and 2.5 $\mathrm{m}$ in the $x, y$, and $z$ directions, respectively, and the contaminant fate and plume are simulated based on these grid blocks. Three remediation periods, namely, 5, 10, and 15 years, represent the short, medium, and long durations, respectively. The main contaminants of this site are petro-

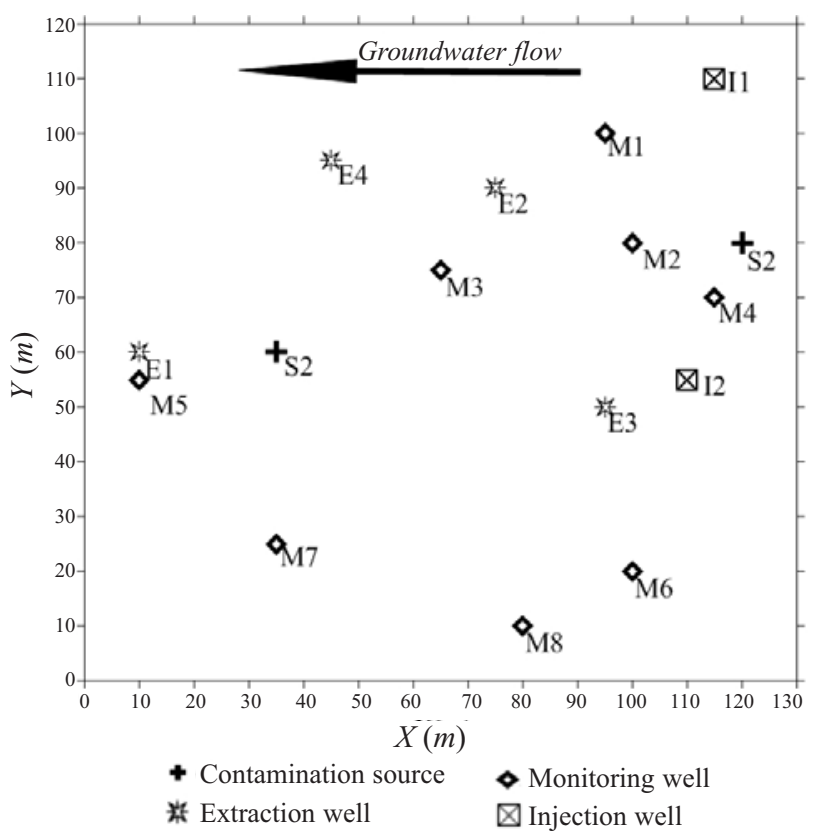

Fig. 1. Well configuration. 
leum products, as per these two sources. Benzene is considered the representative substance of these contaminants because of its high toxicity to the environment and carcinogenicity to humans. The concentrations of other petroleum pollutants fall below the environmental standard as long as the benzene concentrations meet the standard [29]. Therefore, benzene concentration is the sole contaminant concentration considered in all monitoring wells in the current study. The lower bounds of both the extraction and injection rates are set to zero according to the site characteristics, whereas the upper bounds do not exceed $100 \mathrm{~m}^{3} / \mathrm{h}$. The choice of statistical weight significantly influences the optimization results. This study then applies the mean weight analysis method to assign 10 variables. In the various scenarios, the set environmental standards range from $50 \mu \mathrm{g} / \mathrm{L}$ to $150 \mu \mathrm{g} / \mathrm{L}$. The input parameters for the simulated model are presented in Table 1.

\section{Optimization Analysis}

Optimization can be influenced directly by remediation periods, environmental standards, and the given total pumping rates. In other words, these three factors produce various remediation scenarios. The optimal results are analyzed using the single factor method, and $\widehat{d}_{k}^{-}$level indicates that different remediation effects may rely directly on the three factors. Fig. 2 displays the influence of these three factors on the optimal results. Subfigures (a), (b), and (c) correspond to the $\widehat{d}_{k}^{-}$levels in eight monitoring wells when the environmental standard is $100 \mu \mathrm{g} / \mathrm{L}$ and the given total pumping rate is $150 \mathrm{~m}^{3} / \mathrm{h}$. The remediation durations are 5, 10, and 15 years. A high $\widehat{d}_{k}^{-}$level indicates low benzene concentration in the $k$ th monitoring well. Moreover, the $\widehat{d}_{k}^{-}$ values evidently change over 5 years to 10 years of remediation in all eight monitoring wells. Almost all of the $\hat{d}_{k}^{-}$ values increase; as a result, the benzene concentrations decrease significantly because of the strong remediation effect. However, $\widehat{d}_{k}^{-}$values differ only slightly between 10 years to 15 years of remediation. During the short remediation period, the remediation effect is less significant than in the medium and long remediation durations. Increased $\widehat{d}_{k}$ levels enhance the remediation prospects of wells M1, M3, and M8 relative to the other wells without remediation variations. Figs. 2(d-f) display the strict and relaxed $\widehat{d}_{k}^{-}$levels (i.e., 50, 100, and $125 \mu \mathrm{g} / \mathrm{L}$ ). These three subfigures present the constraints of five years of remediation and $200 \mathrm{~m}^{3} / \mathrm{h}$ total pumping rate. Although all $\hat{d}_{k}^{-}$levels increase considerably, the environmental standard increases as well. Additionally, the accelerated enhancement of the environmental standard exceeds that of the $\widehat{d}_{k}{ }_{k}^{-}$level. Thus, the concentrations in most of the wells increase in the process. Furthermore, the benzene concentrations in wells M3 and M6 do not vary significantly. However, these wells meet the environmental standard, which suggests that the concentrations in both wells are close to zero. Therefore, wells M3 and M6 display high remediation levels under these scenarios, followed by wells M1 and M8 and wells M2 and
Table 1. Part of input parameters for the simulation model.

\begin{tabular}{|c|c|c|}
\hline Parameter & Value & Unit \\
\hline Residual water saturation & 0.10 & - \\
\hline Residual oil saturation & 0.20 & - \\
\hline Residual gas saturation & 0.10 & - \\
\hline $\begin{array}{l}\text { Permeability of sandy soil in } x, y \text {, and } \\
z \text { direction }\end{array}$ & 29000 & MD \\
\hline $\begin{array}{l}\text { Permeability of clay till in } x, y \text {, and } z \\
\text { direction }\end{array}$ & 195 & MD \\
\hline $\begin{array}{l}\text { Permeability of silty clay in } x, y \text {, and } z \\
\text { direction }\end{array}$ & 380 & MD \\
\hline Porosity of sandy soil & 0.35 & - \\
\hline Porosity of till & 0.30 & - \\
\hline Porosity of silty clay & 0.53 & - \\
\hline NAPL/water interfacial tension & 45 & Dyne/cm \\
\hline NAPL density & 0.713 & $\mathrm{~g} / \mathrm{cm}^{3}$ \\
\hline Longitudinal dispersivity of sandy soil & 5 & $\mathrm{~m}$ \\
\hline Longitudinal dispersivity of clay till & 5 & $\mathrm{~m}$ \\
\hline Longitudinal dispersivity of silty clay & 5 & $\mathrm{~m}$ \\
\hline Transverse dispersivity of sandy soil & 0.5 & $\mathrm{~m}$ \\
\hline Transverse dispersivity of clay till & 0.5 & $\mathrm{~m}$ \\
\hline Transverse dispersivity of silty clay & 0.5 & $\mathrm{~m}$ \\
\hline Hydraulic gradient & 0.003 & $\mathrm{~m} / \mathrm{m}$ \\
\hline $\begin{array}{l}\text { NAPL/water partition coefficient of } \\
\text { benzene }\end{array}$ & 0.00203 & - \\
\hline $\begin{array}{l}\text { NAPL/water partition coefficient of } \\
\text { E-benzene }\end{array}$ & 0.000173 & - \\
\hline $\begin{array}{l}\text { NAPL/water partition coefficient of } \\
\text { toluene }\end{array}$ & 0.000594 & - \\
\hline $\begin{array}{l}\text { NAPL/water partition coefficient of } \\
\text { xylenes }\end{array}$ & 0.000175 & - \\
\hline Benzene solubility & 1750 & $\mathrm{mg} / \mathrm{L}$ \\
\hline Ethylbenzene solubility & 152 & $\mathrm{mg} / \mathrm{L}$ \\
\hline Toluene solubility & 535 & $\mathrm{mg} / \mathrm{L}$ \\
\hline Xylenes solubility & 175 & $\mathrm{mg} / \mathrm{L}$ \\
\hline Time step at $\mathrm{t}=0$ & 0.101 & day \\
\hline Maximum time step size & 10 & day \\
\hline Tolerance for concentration change & 0.001 & - \\
\hline
\end{tabular}

M7, which show relatively poor levels. When total pumping rate varies, performance can change under a medium period (10 years) and a relaxed environmental standard (Figs. 2(g-i)). The given total pumping rates in these three subfigures are 100,150 , and $200 \mathrm{~m}^{3} / \mathrm{h}$. In all three of these scenarios, almost all of the monitoring wells are effective. 
(a)

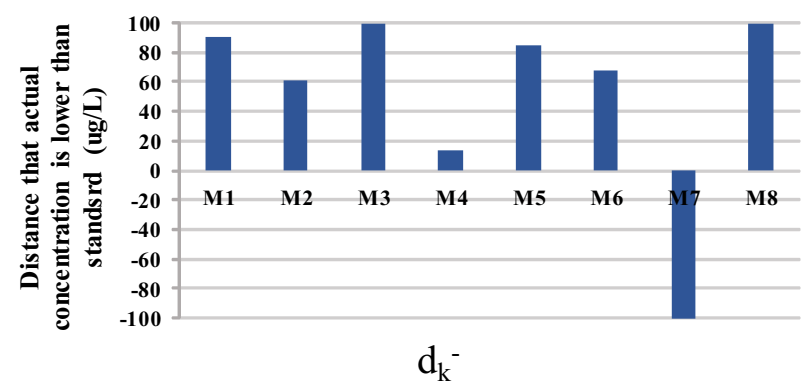

(b)

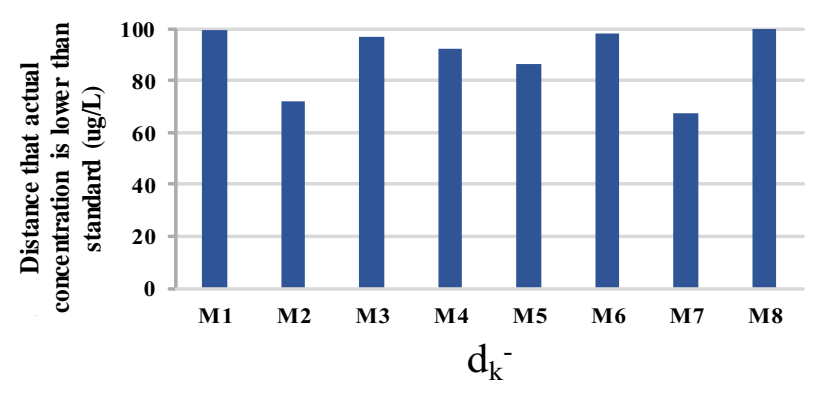

(c)

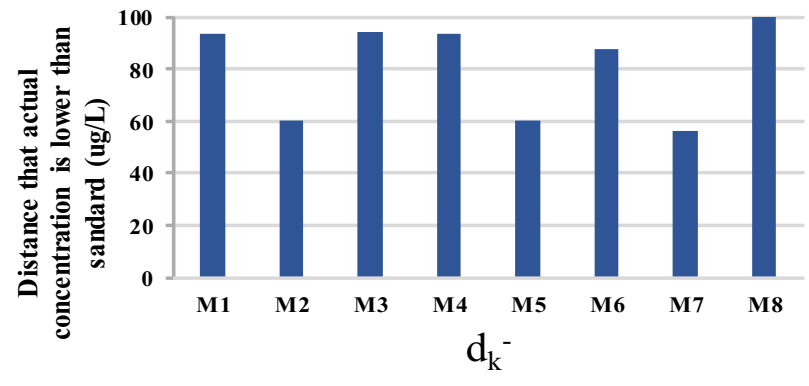

(d)

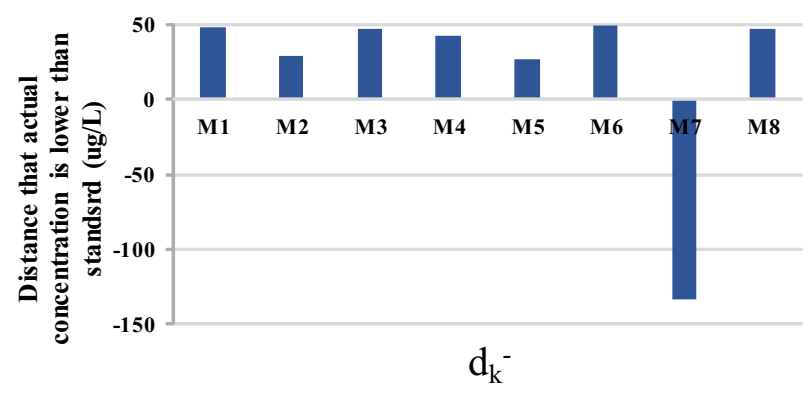

(e)

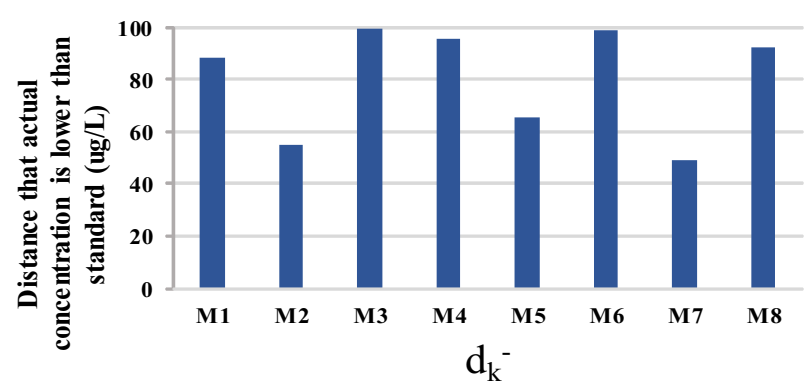

(f)

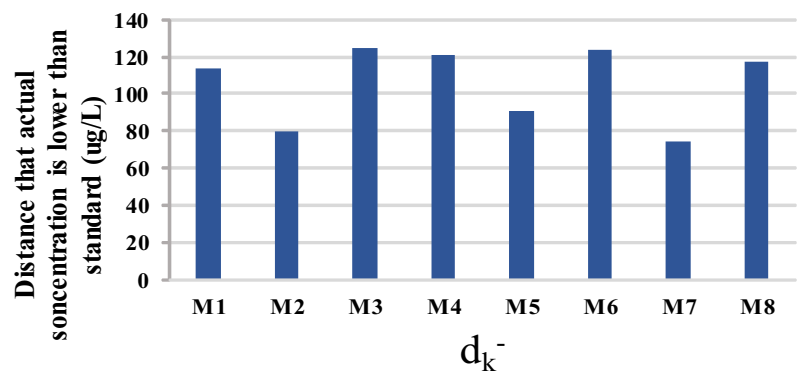

(g)

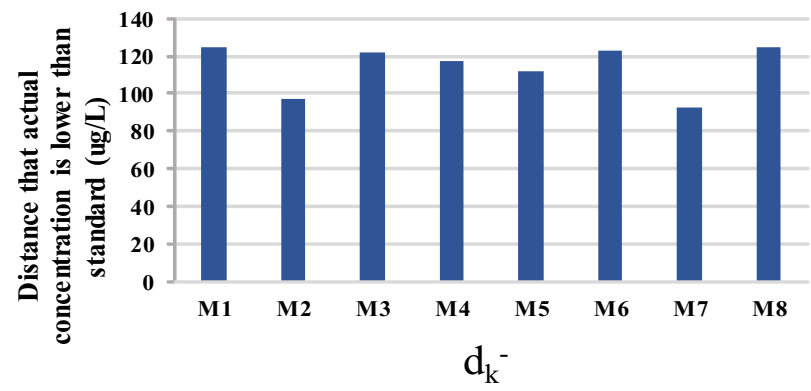

(h)

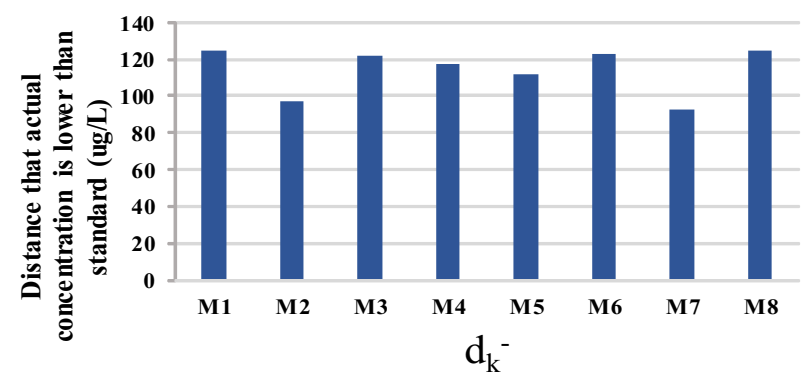

(i)

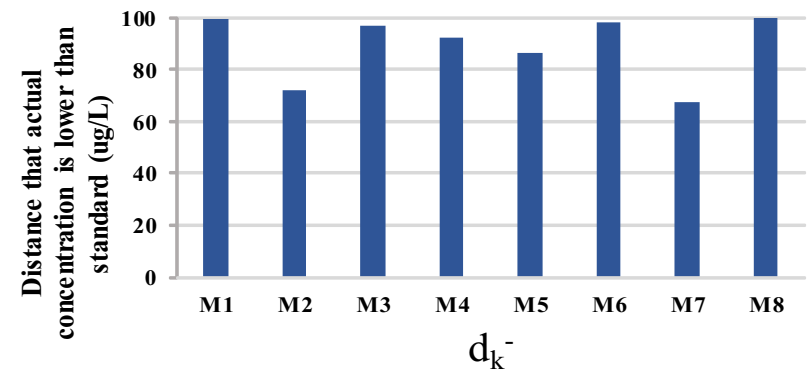

Fig. 2. Levels of $\widehat{d}_{k}^{-}$for eight monitoring wells. 
Furthermore, the $\widehat{d}_{k}^{-}$level of Fig. 2(i) drops minimally. This figure displays the highest total pumping rate among the three scenarios. The drop in $\widehat{d}_{k}^{-}$level is attributed to the relatively long remediation period and the relaxed standard, which reduce total pumping rate. Thus, a high total pumping rate does not always produce desirable outcomes. Moreover, the sign of $\widehat{d}_{k}^{-}$has been negative more than once. In other words, the optimized $\widehat{d}_{k}^{-}$is zero and $\widehat{d}_{k}{ }^{+}$is positive. Well M7 contains much benzene; therefore this well is particularly sensitive to short remediation periods. Furthermore, this well in particular should be monitored to avoid exceeding the set concentration in practice.

The benzene concentration in each monitoring well can intuitively reflect the predicted remediation results, unlike the $\widehat{d}_{k}^{-}$levels. Fig. 3 presents the benzene concentrations in eight monitoring wells under nine simulated scenarios to further explore the effect of goal programming on the optimization solutions. Each result is compared with the solution of the basic model that did not apply goal programming [30]. In the figure, the short black line in each well represents the original concentration. Single-factor analysis is also introduced. The first three subfigures describe the scenarios with the short, medium, and long remediation durations when the concentration standard is $100 \mu \mathrm{g} / \mathrm{L}$ and the given total pumping rate is $200 \mathrm{~m}^{3} / \mathrm{h}$. In this case, the predicted concentrations in the eight wells are considerably lower during the 10-year period than those during the fiveyear period. This result indicates that a 10 -year remediation period is better than a five-year one. The concentrations in some of the wells (i.e., M3 and M4) decrease from 10 years to 15 years. Nonetheless, the concentrations in some of the other wells increase slightly (i.e., M1, M7, and M8), whereas that in well M2 remains constant. However, the concentrations in wells M5 and M6 are high. Furthermore, the additional five years do not enhance remediation effect. The number axis on the right corresponds to the degree of original results. With goal programming, the predicted benzene concentrations are significantly lower than the original concentrations predicted under a 10 -year period. The concentrations at wells M5 and M6 are most strongly reduced within the five-year period. During the 10- and 15-year periods, the concentrations at wells M1 and M2 decrease rapidly. All six concentrations decreased from nearly $100 \mu \mathrm{g} / \mathrm{L}$ to $20 \mu \mathrm{g} / \mathrm{L}$ or to 0 . The three subfigures in the center of Fig. 2 depict the predicted concentrations under the short (five years) and medium remediation periods, giving a total pumping rate of $150 \mathrm{~m}^{3} / \mathrm{h}$. Moreover, the environmental standard constraints in the three central subfigures are 50, 100 , and $150 \mu \mathrm{g} / \mathrm{L}$. Subfigures (d) and (e) indicate that the concentrations in all monitoring wells increase with the relaxation of the standard constraint. However, the concentration in each monitoring well drops when the environmental standard continues to increase to $150 \mu \mathrm{g} / \mathrm{L}$. Despite the significant decrease in concentrations in wells M5 and M6, approximately half of the wells display benzene concentrations that are higher than the original ones predicted without goal programming under the same scenario. The concentration in well M7 remains constant at $200 \mu \mathrm{g} / \mathrm{L}$ under all three scenarios, which exceeds the environmental standard constraint. Thus, well M7 is unsuitable for these three scenarios. These scenarios also do not improve significantly under goal programming. The final three subfigures illustrate the predicted concentrations given three total pumping rates. The environmental standard is set to $50 \mu \mathrm{g} / \mathrm{L}$ and the remediation duration to 15 years. In Subfigures (g), (h), and (i), the given total pumping rates are 100, 150, and $200 \mathrm{~m}^{3} / \mathrm{h}$, respectively. The concentrations in most of the wells depicted in Subfigure (h) are lower than those in Subfigure (g). In Subfigure (i), however, the concentrations vary slightly when the given total rate shifts between 150 and $200 \mathrm{~m}^{3} / \mathrm{L}$. We can infer that the additional $50 \mathrm{~m}^{3} / \mathrm{h}$ does not improve the results. Well M5 has the highest predicted level of benzene concentration at values of 40, 69, and 79 $\mu \mathrm{g} / \mathrm{L}$, which are close to or even beyond the environmental standard $(50 \mu \mathrm{g} / \mathrm{L})$. This finding indicates that well M5 is sensitive to strict environmental constraints and should be monitored intentionally. When goal programming is introduced, the original and predicted concentrations are effective. Moreover, the original concentrations in some wells are significantly higher than the ones predicted with goal programming. However, the original low concentrations in the other wells do not surpass the predicted concentrations under goal programming. Well M5 alone increases slightly. Therefore, remediation with goal programming is efficient in these scenarios. Two conclusions can then be drawn based on this figure. First, the remediation period most significantly influences the remediation effect among the three main factors. In particular, the 10 -year remediation period is effective. Second, the remediation effect is enhanced by goal programming relative to the original one.

The extraction and injection rates in each extraction well and injection well also reflect the optimization results. These rates are related to operating cost. The total extraction rate must be equal to the total injection rate to maintain a stable hydraulic gradient. In Formula (9h), $\widehat{r}^{+}$and $\widehat{r}^{-}$cause an imbalance between the total extraction and total injection rates in the optimization results of Model (8). Therefore, the difference between the total extraction and total injection rates must be considered. If the total extraction rate exceeds the total injection rate, a supplement of external clean water must be injected into the subsurface to ensure that groundwater can flow directly into the plume. Conversely, the additional injected water is removed. The nine pie charts (Fig. 4) show the disequilibrium between the total extraction and total injection rates. The scenarios in four pie charts (Figs. 4(a), 4(g), 4(h), and 4(i)) are simulated under a five-year remediation duration. Moreover, the total extraction rates are significantly higher than the total injection rates. The injection rate remains at $20 \%$ and may even drop to $0 \%$. When the remediation duration is long at 15 years, two predictions are encountered. The absolute balance is below the $50 \mu \mathrm{g} / \mathrm{L}$ environmental standard and the $150 \mathrm{~m}^{3} / \mathrm{h}$ total pumping rate, as shown in Chart (e). Chart (c) follows with $45 \%$ of the total injection rate and $55 \%$ of the total extraction rate. In the other two scenarios, the total injection rate remains significantly lower than the total extraction rate. The results vary significantly under a 
(a) Benzene conentration (ug/L)
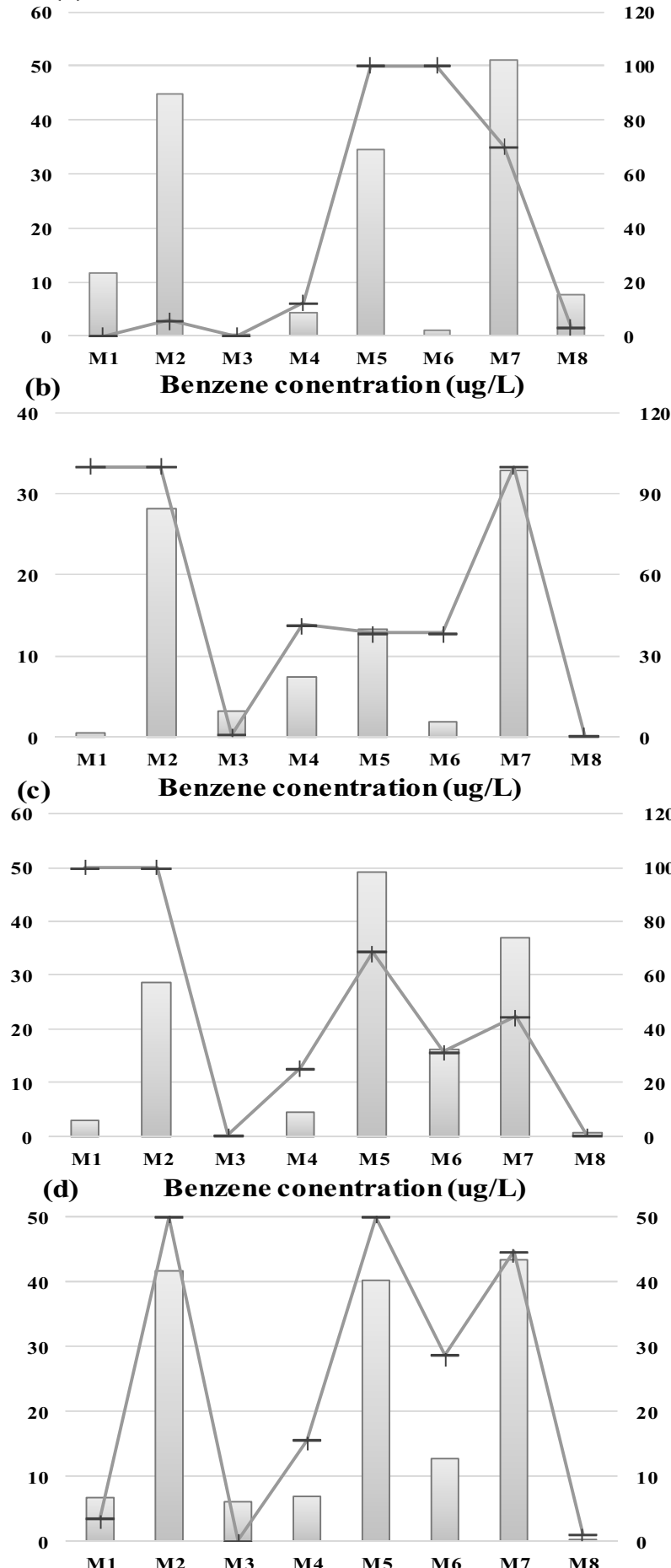

(e) Benzene conentration (ug/L)
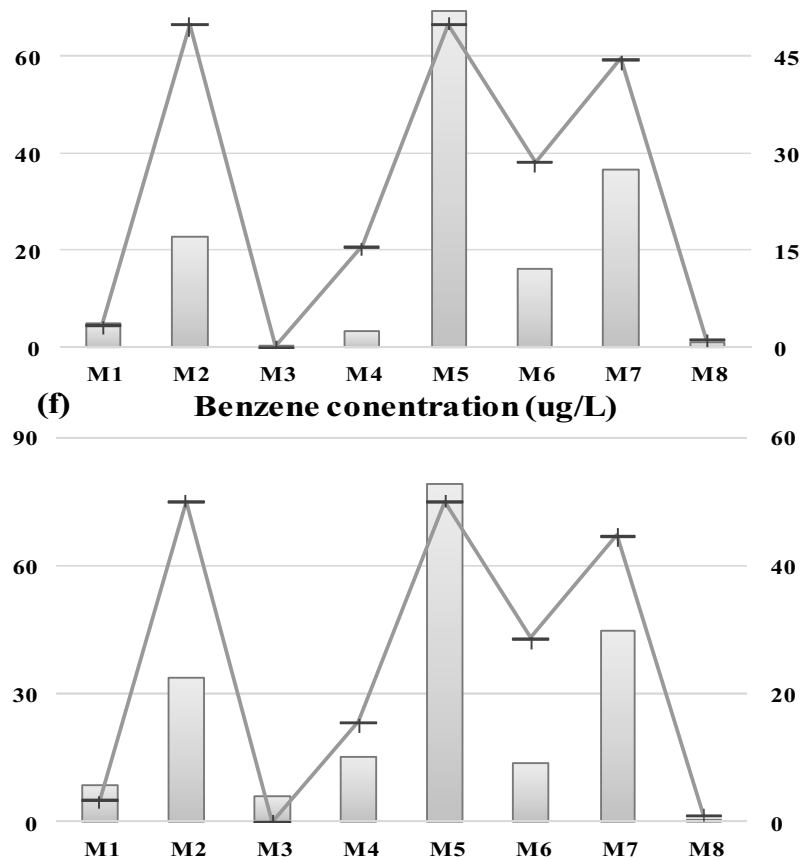

(g) Benzene conentration $(u g / L)$
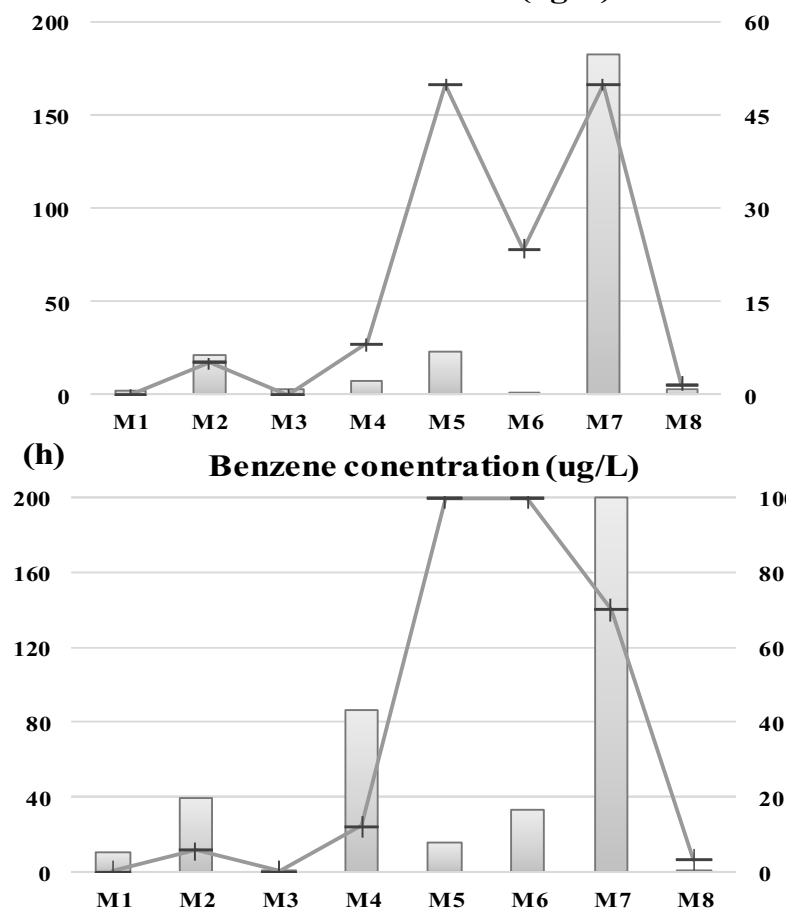

Benzene conentration $(\mathrm{ug} / \mathrm{L})$

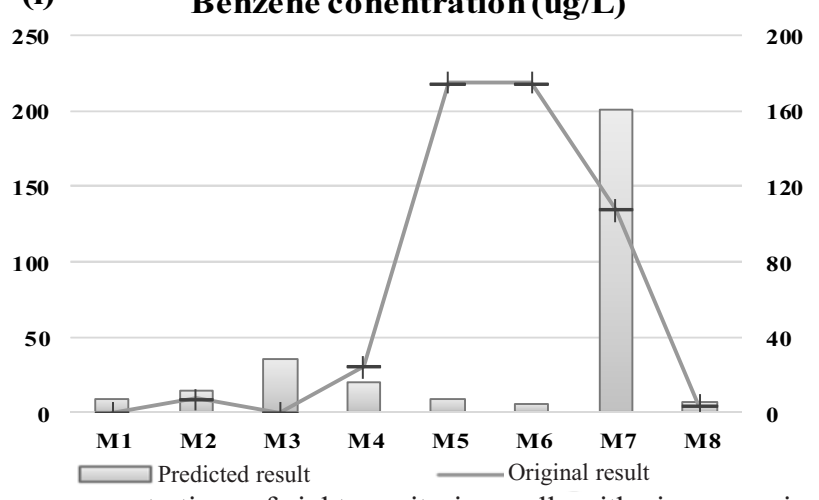

Fig. 3. Original and predicted benzene concentrations of eight monitoring wells with nine scenarios. 
a)

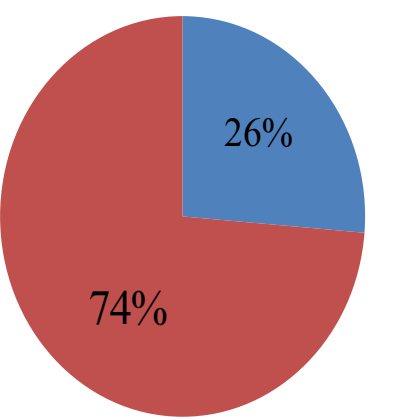

b)

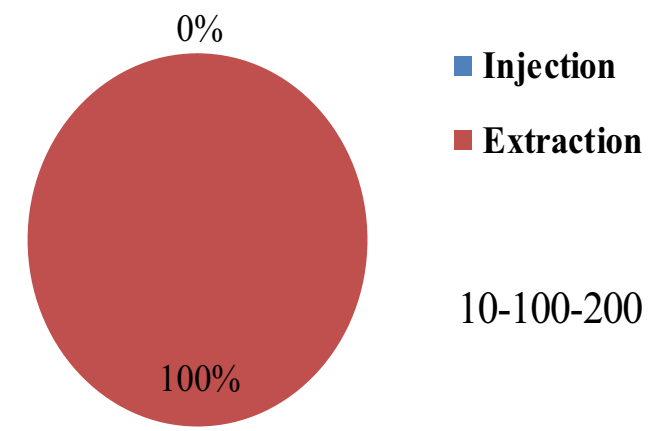

c)

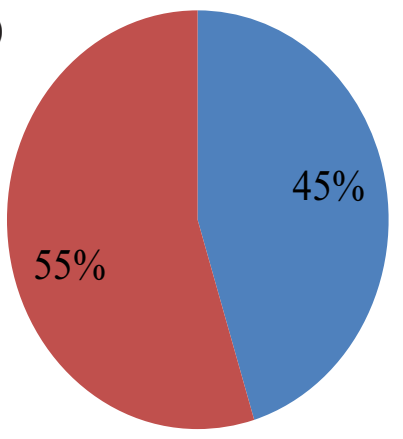

d)

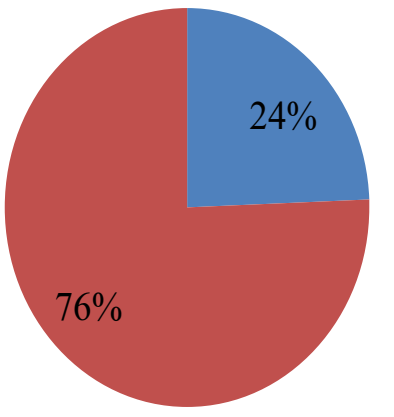

Injection

Extraction

5-100-200 e)

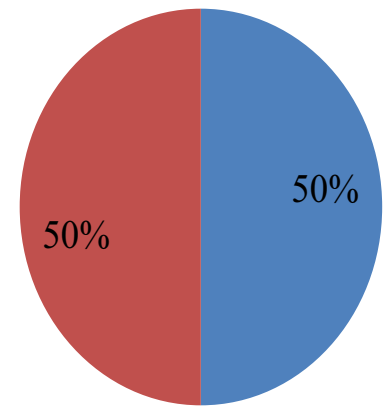

f)

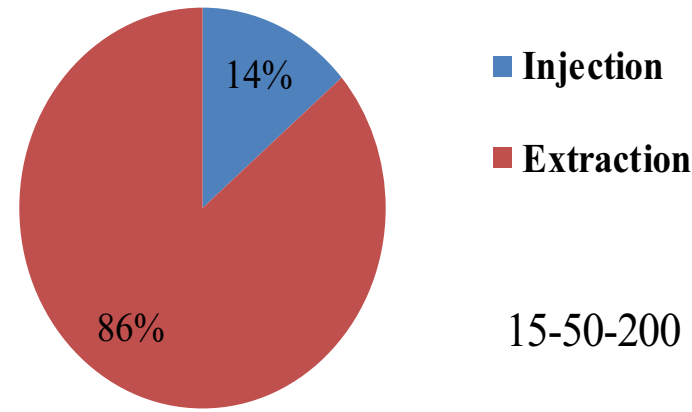

g)

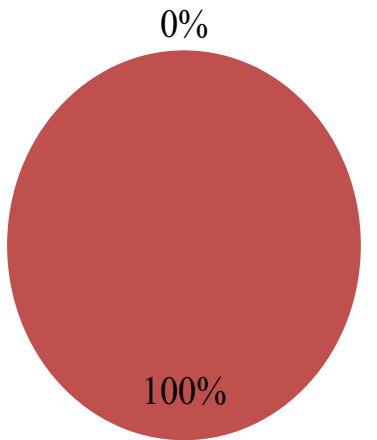

h)

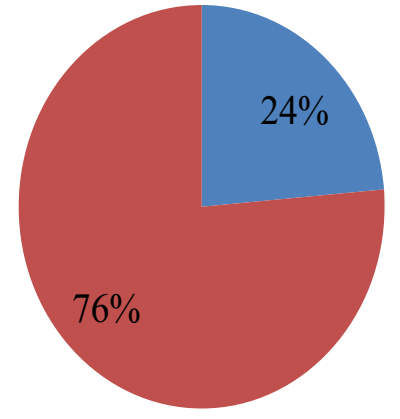

- Injection

- Extraction

$15-50-150$
- Injection

- Extraction

5-50-150

Extraction

5-100-150
Injection
Injection

Extraction

15-100-200

- Injection

Extraction

15-50-100

i)

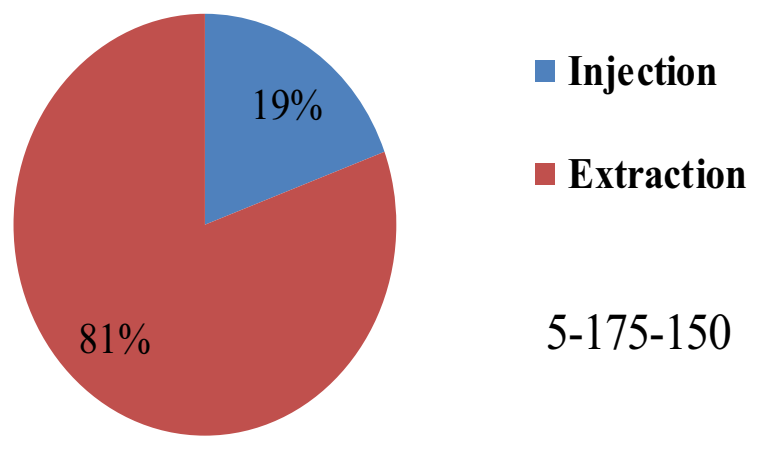

Fig. 4. Proportions of total injecting and extracting rates under nine scenarios. 
strict environmental standard $(50 \mu \mathrm{g} / \mathrm{L})$ because of the different remediation periods and given total pumping rates. The percentage of the total injection rate thus increases from $0 \%$ to $50 \%$. We can obtain similar results by comparing the different remediation periods and environmental standards under a constant given total pumping rate. In conclusion, the total injection rate is lower than the total extraction rate in most of the scenarios. With this situation in mind, external clean water should therefore be imported to the system to maintain hydraulic balance.

a)

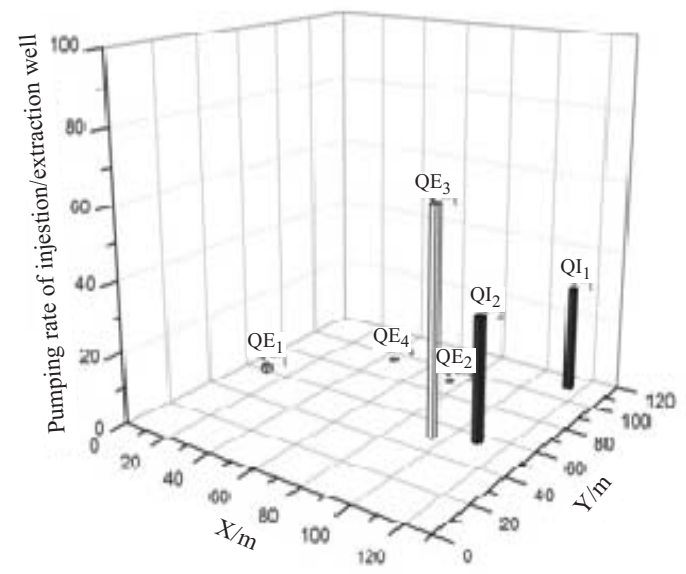

c)

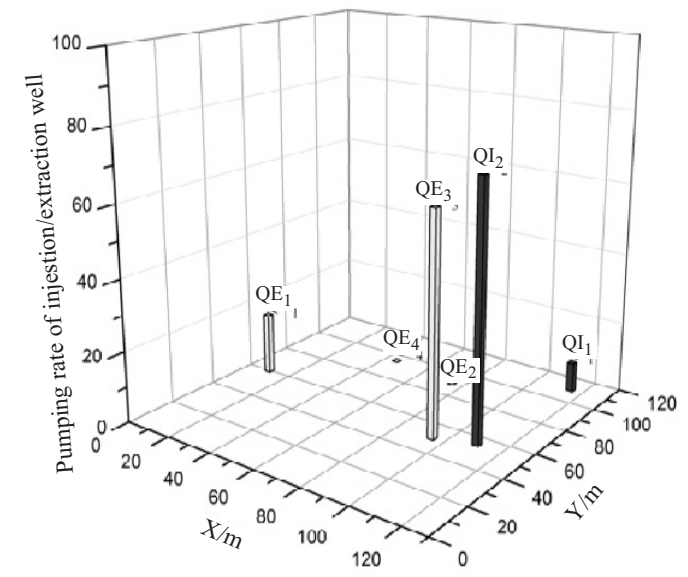

e)

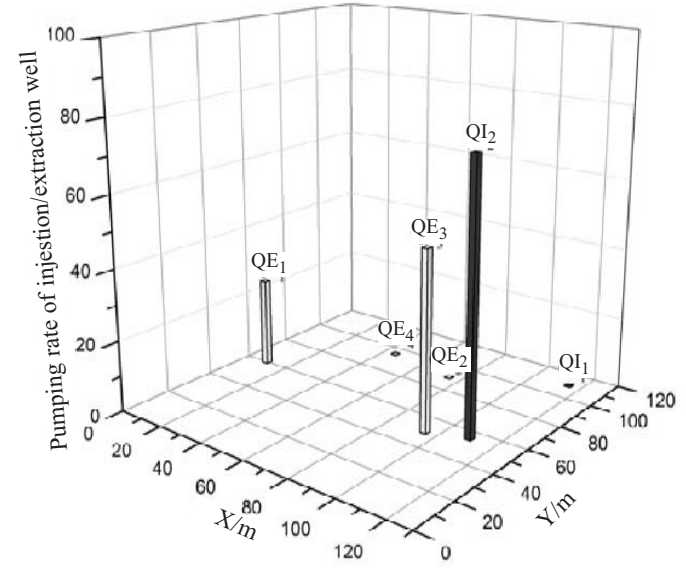

However, the importation of external water induces a series of problems, with a major one being economic cost. Therefore, this solution is inappropriate if decision-makers are focused on financial burden. If $\widehat{r}^{+}$and $\widetilde{r}^{-}$are set to zero in Formula (9h), the total extraction rate is equal to the total injection rate, thereby eliminating the concern regarding extra cost. Fig. 5 presents the extraction and injection rates in each well under six scenarios. Four extraction and two injection wells are positioned according to their coordinates on a horizontal plane that is composed of $\mathrm{x}$ - and $\mathrm{y}$-axes.

b)

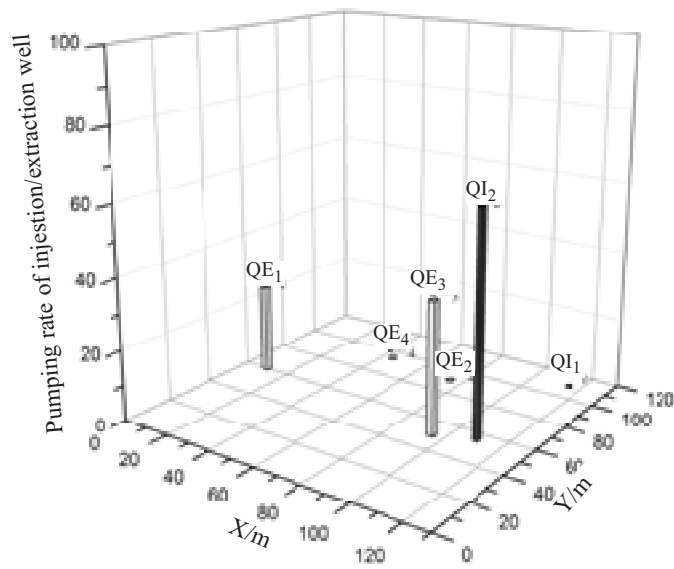

d)

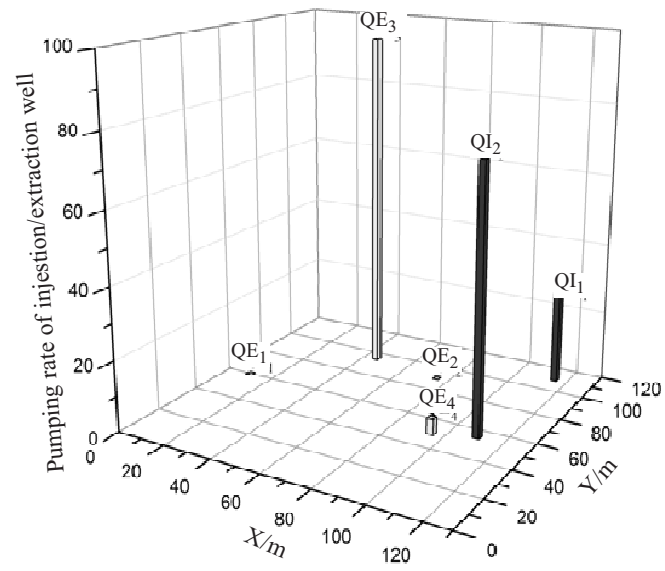

f)

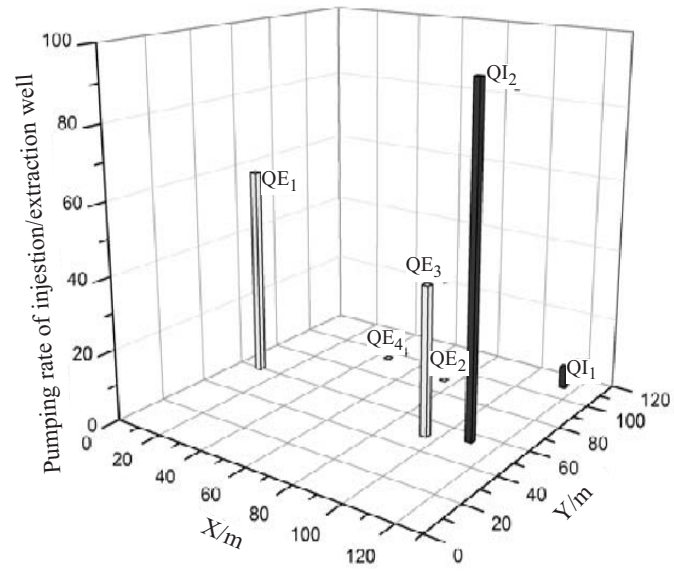

Fig. 5. Injecting and extracting rates of six pumping wells under six scenarios. 
The vertical axis in the $\mathrm{z}$ direction represents the levels of either the extraction or the injection rate. Subfigures (a) and (b) illustrate six pumping rates within the five- and 10-year remediation periods, a $100 \mu \mathrm{g} / \mathrm{L}$ environmental standard, and a given total pumping rate of $150 \mathrm{~m}^{3} / \mathrm{h}$. In these scenarios, wells I1, I2, E1, and E3 vary considerably. Wells E2 and E4 are nearly inoperable, and the injection rate of well I1 at the five-year period has been transferred to well I2 at the 10 year period. Furthermore, approximately less than half of the extraction rate of well E3 in Subfigure (a) shifts to well E1 in subfigure (b). Under these two scenarios, three of the pumping wells are operational, whereas the other three are inoperable. The total pumping rate is constant at approximately $120 \mathrm{~m}^{3} / \mathrm{h}$, which controls cost. The constant scenario constraints in Subfigures (c) and (d) are a 10-year remediation duration and a given total pumping rate of 200 $\mathrm{m}^{3} / \mathrm{h}$. The corresponding environmental standards are 50 and $200 \mu \mathrm{g} / \mathrm{L}$, and they represent the strict and relaxed standard constraints. The two injection rates change slightly, whereas the extraction rates in wells E1, E3, and E4 vary significantly. Under the strict standards, well E1 generates part of the extraction rate; however, this rate is unused under the relaxed standard. Well E3 typically accounts for most of the extraction rates. Moreover, well E3 is almost fully operational under a relaxed standard. This well is the most significant among the extraction wells. The scenario constraints of Fig. 5 (e) and (f) differ in terms of given total pumping rate; that of the former is $150 \mathrm{~m}^{3} / \mathrm{h}$ and that of the latter is $200 \mathrm{~m}^{3} / \mathrm{h}$. The environmental standard is $125 \mu \mathrm{g} / \mathrm{L}$, and the remediation period is five years. Thus, the predicted total pumping rate increases to some extent. Moreover, wells I2, E1, and E3 comprise the main PAT system. Well I2 generates most of the injection rate, whereas well I1 accounts for a small part of it. The extraction rate at well E3 is higher than that at well E1, as indicated in subfigure (e). In Subfigure (f), the increase in the predicted total pumping rate is transferred to well $\mathrm{E} 1$. As a result, the extraction rate of well E1 is higher than that of well E3. The scenarios above suggest that wells I2, E1, and E3 are important in the PAT system. The extraction rates of wells E2 and E4 usually remain low, and these wells are inoperable in most scenarios. Well I2 is more important than well I1 in the injection of treated water because the injection rate in the former is generally significantly higher than that in the latter. The difference in the importance of these pumping wells may be attributed to the property of benzene, groundwater flow direction, and well distribution. Finally, these factors influence one another in interaction.

\section{Conclusions}

Goal programming is effective and highly efficient as a conventional tool to address multi-objective problems. In most scenarios, the predicted concentrations under goal programming are much lower than the original predicted concentrations. The optimal strategies can also be directly influenced by remediation periods, environmental standards, and given total pumping rates. In addition, the short remediation duration is worse than the medium and long durations. Nonetheless, wells M3 and M6 perform ideally in a short remediation period because the benzene concentrations are almost zero. The remediation areas around wells M1, M3, and M8 generally have good remediation prospects. Moreover, wells I2, E1, and E3 are significant in the PAT system. Well M7 is sensitive to short remediation periods and must be managed to control concentration. Well M5 is sensitive to strict environmental constraints and should be monitored intentionally. Furthermore, these factors influence one another in interaction. Finally, high total pumping rates do not always generate favorable outcomes, and a long remediation period is unnecessary. Therefore, these three factors should be spontaneously considered in a general framework.

In the future, advanced methods should be integrated with goal programming to improve the optimal models. Moreover, parameter uncertainties in the models should be considered in optimal design. Finally, the technological and environmental standards in practical engineering may involve additional factors aside from the aforementioned constraints. Therefore, future studies should introduce additional constraints to improve the models.

\section{Acknowledgements}

This research was supported by the China National Funds for Excellent Yong Scientists (51222906), the National Natural Science Foundation of China (41271540), the Program for New Century Excellent Talents in University of China (NCET-13-0791), and Fundamental Research Funds for Central Universities.

\section{References}

1. COMPERNOLLE T., VAN PASSEL S., LEBBE L. The value of groundwater modeling to support a pump and treat design. Ground Water Monit. R. 33, 111, 2013.

2. KO N.Y., LEE K.K. Design of effective remediation system in a contaminated aquifer by controlling constraints. Geosci. J. 13, 415, 2009.

3. KO N.Y., LEE K.K. Information effect on remediation design of contaminated aquifers using the pump and treat method. Stoch. Env. Res. Risk A. 24, 649, 2009.

4. MOUSSAVI G., KHOSRAVI R., FARZADKIA M. Removal of petroleum hydrocarbons from contaminated groundwater using an electrocoagulation process: Batch and continuous experiments. Desalination 278, 288, 2011.

5. GULER C., KAPLAN V., AKBULUT C. Spatial distribution patterns and temporal trends od heavy-metal concentrations in a petroleum hydrocarbon-contaminated site: Karaduvar coastal aquifer (Mersin, SE Turkey). Environ. Earth S. 70, 943, 2013.

6. PARK Y.C., JEONG J.M., EOM S.I., JEONG U.P. Optimal management design of a pump and treat system at the industrial complex in Wonju, Korea. Geosci. J. 15, 207, 2011.

7. MONDAL A., ELDHO T.I., RAO V.V.S.G. Multiobjective groundwater remediation system design using coupled finite-element model and nondominated sorting genetic algorithm II. J. Hydrol. Eng. 15, 350, 2010. 
8. CHANG L.C., CHU H.J., HSIAO C.T. Optimal planning of a dynamic pump-treat-inject groundwater remediation system. J. Hydrol. 342, 295, 2007.

9. MATOTT L.S., RABIDEAU A.J., CRAIG J.R. Pump-andtreat optimization using analytic element method flow models. Adv. Water Resour. 29, 760, 2006.

10. BAU D.A., MAYER A.S. Data-worth analysis for multiobjective optimal design of pump-and-treat remediation systems. Adv. Water Resour. 30, 1815, 2007.

11. HE L., HUANG G.H., LU H.W., ZENG G.M. Optimization of surfactant-enhanced aquifer remediation for a laboratory BTEX system under parameter uncertainty. Environ. Sci. Technol. 42, 2009, 2008.

12. YANG Q., HE L., LU H.W. A multiobjective optimisation model for groundwater remediation design at petroleum contaminated sites. Water Resour. Manag. 27, 2411, 2013.

13. CHENINI I., KHEMIRI S. Evaluation of groundwater quality using multiple linear regression and structural equation modeling. Int. J. Environ. S. Tech. 6, 509, 2009.

14. HANRAHAN G., GARZA C., GARCIA E., MILLER K. Experimental design and response surface modeling: a method development application for the determination of reduced inorganic species in environmental samples. J. Environ. Inform. 9, 71, 2007.

15. HE L., HUANG G.H., LU H.W. A stochastic optimization model under modeling uncertainty and parameter certainty for groundwater remediation design-Part I. Model development. J. Hazard. Mater. 176, 521, 2010.

16. YAN S.Y., MINSKER B. Optimal groundwater remediation design using an adaptive neural network genetic algorithm. Water Resour. Res. 42, 1, 2006.

17. BAU D.A., MAYER A.S. Stochastic management of pumpand-treat strategies using surrogate functions. Adv. Water Resour. 29, 1901, 2006

18. HE L., HUANG G.H., ZENG G.M., LU H.W. An integrated simulation, inference, and optimization method for identifying groundwater remediation strategies at petroleumcontaminated aquifers in western Canada. Water Res. 42, 2629, 2008

19. FAN X., HE L., LU H.W., LI J. Environmental- and healthrisk-induced remediation design for benzene-contaminated groundwater under parameter uncertainty: A case study in Western Canada. Chemosphere 111, 604, 2014.
20. BRAVO M., GONZALEZ I. Applying stochastic goal programming: A case study on water use planning. Eur. J. Oper. Res. 196, 1123, 2009.

21. NIDUMOLU U.B., KEULEN H.V., LUBBERS M., MAPFUMO A. Combining interactive multiple goal linear programming with an inter-stakeholder communication matrix to generate land use options. Environ. Model. Softw. 22, 73, 2007.

22. AL-REFAIE A., DIABAT A. Optimizing convexity defect in a tile industry using fuzzy goal programming. Measurement 46, 2807, 2013.

23. DELICE E.K., GUNGOR Z. Determining design requirements in QFD using fuzzy mixed-integer goal programming: application of a decision support system. Int. J. Prod. Res. 51, 6378, 2013.

24. BEN-AWUAH E., ASKARI-NASAB H., AWUAH-OFFEI K. Production scheduling and waster disposal planning for oil sands mining using goal programming. J. Environ. Inform. 20, 20, 2012.

25. TENNEY C.M., LASTOSKIE C.M., DYBAS M.J. A reactor model for pulsed pumping groundwater remediation. Water Res 38, 3869, 2004

26. MINSKER B.S., SHOEMAKER C.A. Dynamic optimal control of in-situ bioremediation of ground water. J. Water Res.Pl. ASCE 124, 149, 1998.

27. RAO G.T., RAO V.V.S.G., SARMA,V.S. DHAKATE R., SURINAIDU L., MAHESH J., RAMESH G. Hydrogeochemical parameters for assessment of groundwater quality in a river sub-basin. Int. J. Environ. S. Tech. 9, 297, 2012.

28. MIRBAGHERI S.A., MONFARED S.A.H. Pestcide transport and transformation modeling in soil column and groundwater contamination prediction. Int. J. Environ. S. Tech. 6, 233, 2009.

29. KHAMFOROUSH M., BIJAN-MANESH M.J., HATAMI T. Application of the Haug model for process design of petroleum hydrocarbon-contaminated soil bioremediation by composting process Int. J. Environ. S. Tech. 10, 533, 2013.

30. LONGE E.O., ENEKWECHI L.O. Investigation on potential groundwater impacts and influence of local hydrogeology on natural attenuation of leachate at a municipal landfill. Int. J. Environ. S. Tech. 4, 133, 2007. 